\title{
ON THE NATURAL DENSITY OF THE RANGE OF THE TERMINATING NINES FUNCTION
}

\section{ROBERT K. KENNEDY and CURTIS N. COOPER}

\author{
Department of Mathematics and Computer science \\ Central Missourl State University \\ Warrensburg, Missouri 64093 \\ and
}

\section{VLADIMIR DROBOT and FRED HICKLING}

\author{
Department of Mathematics \\ Santa Clara University \\ Santa Clara, CA 95053
}

(Received January 15, 1988 and in revised form June 6, 1988)

ABSTRACT. Noting that the expression $\sum_{t \geqslant 1}\left[\frac{n}{10^{t}}\right]$ gives the number of terminating nines which occur up to $n$ but not including $n$, we will denote the above expression by $t(n)$ and call $t$ the "terminating nines function". The natural density of the set $T=\{t(n): n=1,2,3, \ldots\}$ will be determined.

KEY WORDS AND PHRASES. Digital sums, terminating nines, natural density.

1980 AMS SUBJECT CLASS IFICATION CODES. Primary 11A63, Secondary: none.

\section{INTRODUCTION.}

The number of positive integers in a set $A$, not exceeding $x$, is denoted by $A(x)$. The natural density, $d(A)$, of the set $A$ is defined as

$$
d(A)=\lim _{x+\infty} \frac{A(x)}{x},
$$

provided this limit exists. The determination of the natural density of a given set of positive integers is an important topic in most number theory textbooks and is the subject of much research.

For example, the set of positive integers

$$
N=\{n: s(n) \text { is a factor of } n\},
$$

where $s(n)$ denotes the digital sum of $n$, is the set of Niven numbers [1] and was shown to have a natural density of 0 in [2]. Here, we are interested in a part of the digital surn function. 
It has been shown that

$$
s(n)=n-9 \sum_{t \geqslant 1}^{1}\left[\frac{n}{10^{t}}\right]
$$

where, as usual, the square brackets denote the integral part operator. Noting that the expression

$$
\sum_{t}\left[\frac{n}{10^{t}}\right]
$$

gives the number of terminating nines which occur up to $n$ but not includitg $n$, we will denote $(1.1)$ by $t(n)$ and call the "terminating nines function". The natural density of the set $T=\{t(n): n=1,2,3, \ldots\}$ will be determined in what follows. Note that $T$ does not include every positive integer since, for example, $10 \mathrm{k}$.

\section{NOTATION ANI) TERMINOLOGY.}

In what follows, we will say that the terminating nines function, $t$, has a "jump" of size $k$ at an integer a if $t(a)=t(a-1)+k$. Thus, $t$ has a jump of size $k$ if and only if $a-1$ ends with exactly $k$ nines. To determine the natural density of $T$, we first show that

$$
\lim _{n \rightarrow \infty} \frac{T(t(n))}{t(n)}=\frac{9}{10}
$$

where $T(t(n))$ is the number of members of $T$ not exceeding $t(n)$. To do this, we will count how many integers are missing from set $\{t(1), t(2), \ldots, t(n)\} . \quad$ If $\alpha_{n}$ is the number of these missing integers, then it follows that

$$
T(t(n))=t(n)-\alpha_{n}
$$

3. THE NATURAL DENSITY OF $\mathrm{T}$.

Noting that if $1<a<n$ and $t$ has a jump of size $k$ at $a$, then this jump will produce k-1 missing integers. Moreover, each missing integer is a result of some jump at a for $1<a<n$. Thus, each $1<a<n$, such that $10^{k}$ divides a but $10^{k+1}$ does not divide a, produces $k-1$ missing integers. Hence, $\alpha_{n}$ is the number of terminating 0 's in all integers $1 \leqslant a<n$, minus the number of integers $1 \leqslant a<n$ which end with D. Therefore, since

$$
\alpha_{n}=\sum_{j \geqslant 1}\left[\frac{n}{10^{j}}\right]-\left[\frac{n}{10}\right],
$$

we have that

$$
T(t(n))=\left[\frac{n}{10}\right] .
$$


Using the above, we thus conclude that

which nay be written as

$$
\frac{T(t(n))}{t(n)}=\frac{\left[\frac{n}{10}\right]}{\left[\frac{n}{10}\right]+\left[\frac{n}{10^{2}}\right]+\ldots}
$$

$$
\frac{T(t(n))}{t(n)}=\frac{\frac{n}{10}+0(1)}{\frac{n}{10}+\frac{n}{10^{2}}+\ldots+0(1 \circ \mathrm{log})}
$$

since the denominator is equal $\frac{n}{10}+\frac{n}{10^{2}}+\ldots+0(\log n)$, and the numerator is equal
to $\frac{n}{10}+0(1)$. Thus, to $\frac{\mathrm{n}}{10}+0(1)$. Thus,

$$
\begin{aligned}
& \lim _{n \rightarrow \infty} \frac{T(t(n))}{t(n)}=\lim _{n \rightarrow \infty} \frac{\frac{n}{10}+0(1)}{\frac{n}{10}+\frac{n}{10^{2}}+\ldots+0(\log n)} \\
& =\frac{9}{10} .
\end{aligned}
$$

Letting $x$ be an arbitrary integer, and $y$ be such that

$$
t(y)<x<t(y+1),
$$

we have that $x-t(y)=O(\log x)$ since $x-t(y)$ does not exceed the number of digits in $x$.

Since, $T(x)=T(t(y))$, we have

$$
\frac{T(x)}{x}=\frac{T(t(y))}{x}=\frac{T(t(y))}{t(y)+0(\log x)}
$$

and so, by the above 1 imit, it follows that

$$
\lim _{x \rightarrow \infty} \frac{T(x)}{x}=\frac{9}{10}
$$

Stating this as a theorem we have:

THEOREM 1. Let $T=\{t(n): n=1,2, \ldots\}$ where $t$ is the terminating nines function. Then $\mathrm{d}(\mathrm{T})=\frac{9}{10}$.

\section{GENERALIZATION TO BASE b.}

Finally, it should be noted that the development given above and Theorem 1 can be generalized to any integral base b. If $t_{b}(n)$ denotes the number of terminating b-l's in the base $b$ representation of the sequence of positive integers up to $n$, then we have the following generalization of Theorem 1:

THEOREM 1'. Let $\left\{t_{b}(n): n=1,2, \ldots\right\}$. Then $d(T)=\frac{b-1}{b}$. 
REFERENCES

1. KENNEDY, R., GOODMAN, T. and BEST, C. Matheratical Discovery and Niven Numbers, The MATYC Journal 14 (1980), 21-25.

2. KENNEDY, R. and COOPER, $C$. On the natural density of the Niven numbers, College Math. Journal 15 (1984), 309-312. 


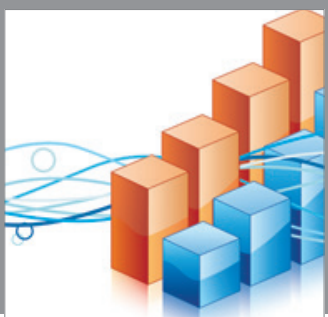

Advances in

Operations Research

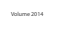

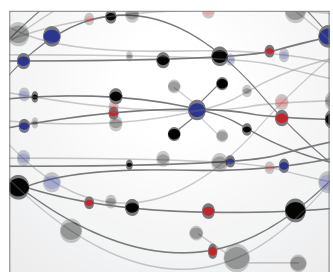

\section{The Scientific} World Journal
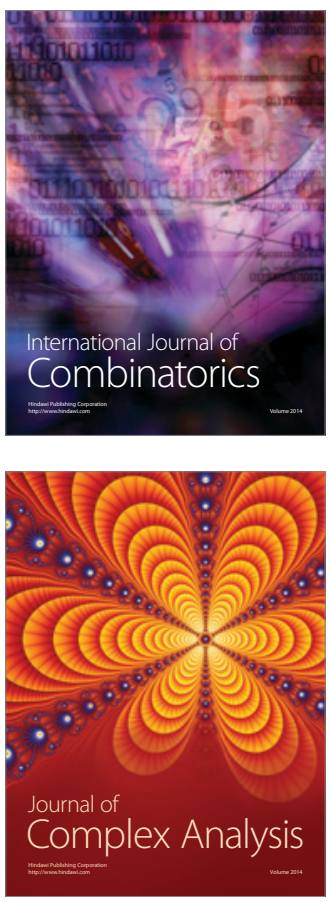

International Journal of

Mathematics and

Mathematical

Sciences
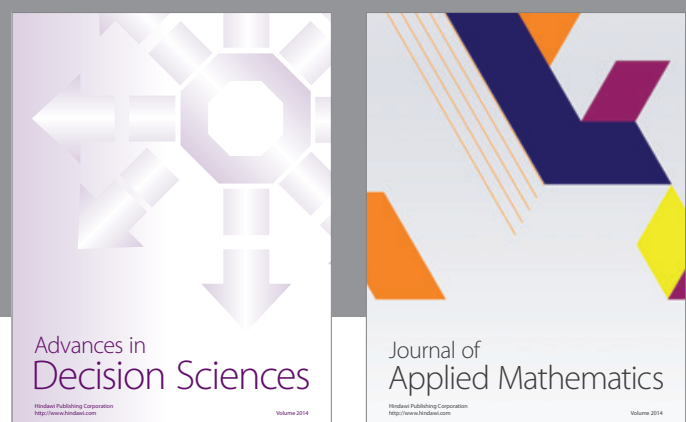

Journal of

Applied Mathematics
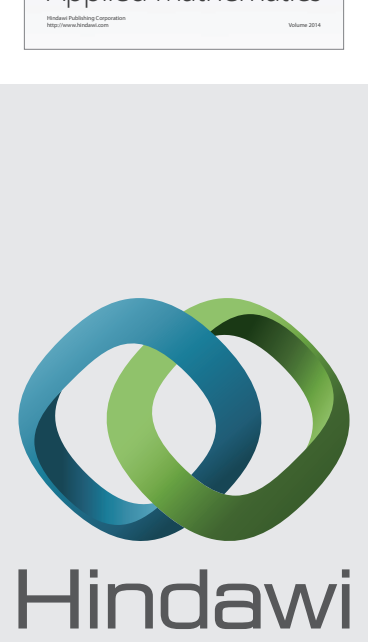

Submit your manuscripts at http://www.hindawi.com
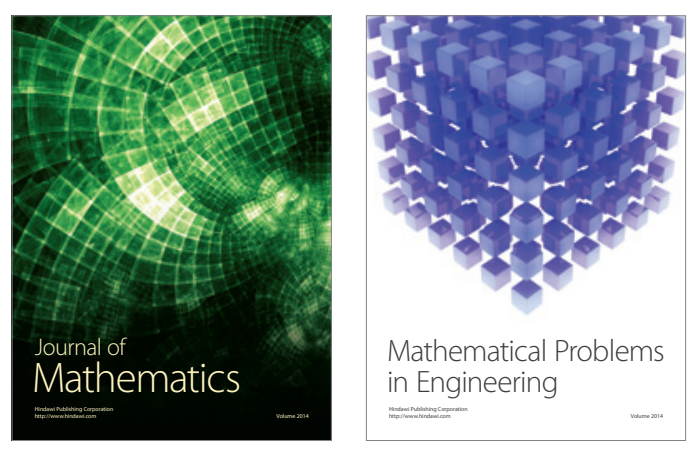

Mathematical Problems in Engineering
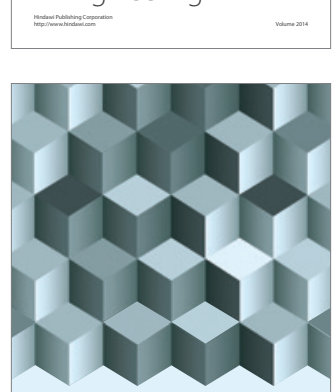

Journal of

Function Spaces
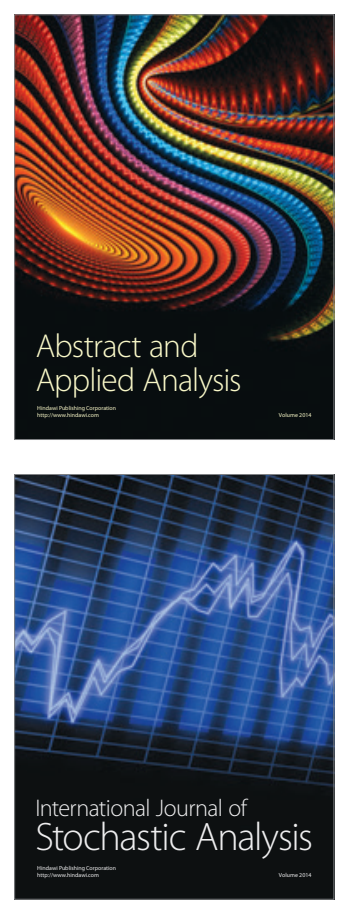

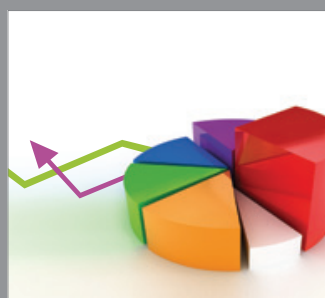

ournal of

Probability and Statistics

Promensencen
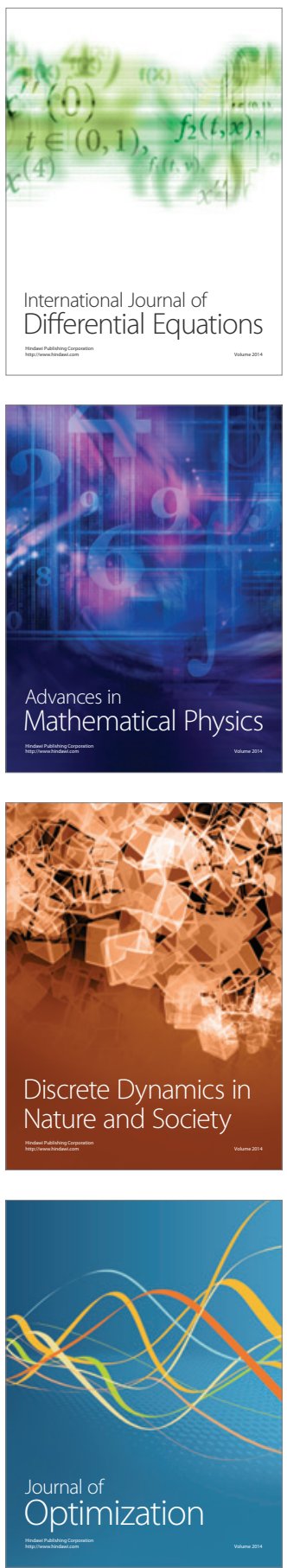